\title{
Modified Adaptive Chirplet Decomposition with Application in ISAR Imaging of Maneuvering Targets
}

\author{
Yong Wang and Yi-Cheng Jiang \\ Research Institute of Electronic Engineering Technology, Harbin Institute of Technology, Harbin 150001, China \\ Correspondence should be addressed to Yong Wang, wangyong6012@hit.edu.cn
}

Received 21 February 2008; Revised 21 July 2008; Accepted 20 October 2008

Recommended by Sharon Gannot

The modified adaptive Chirplet decomposition (MACD) is presented in this paper. It is based on the extension of the traditional Chirplet atoms to the form of the quadratic frequency-modulated signals. The accuracy of the signal decomposition is improved compared with the traditional Chirplet decomposition. A practical algorithm for the parameters estimation of monocomponent MACD atom and the decomposition method based on the CLEAN technique to the multicomponent signals are proposed. Finally, this algorithm is used in the ISAR imaging of maneuvering targets, and the quality of ISAR images improves greatly. The results of real data and simulated data demonstrate the validity of the method proposed.

Copyright (C) 2008 Y. Wang and Y.-C. Jiang. This is an open access article distributed under the Creative Commons Attribution License, which permits unrestricted use, distribution, and reproduction in any medium, provided the original work is properly cited.

\section{INTRODUCTION}

In the inverse synthetic aperture radar (ISAR) imaging of maneuvering targets, the received signals can be considered as multicomponent polynomial phase signals after motion compensation. The parameters of each component should be estimated accurately in order to obtain the high-quality range-instantaneous-Doppler images of the target [1]. For the parameters estimation of multicomponent signals, many methods have been proposed in the past decades, such as the joint time frequency algorithm [2], the CLEAN technique by dechirping, and the RELAX method [3, 4]. But, all of these methods are appropriate to deal with the linear frequencymodulated signals, which can be accepted when the maneuverability of the target is not strong. In practice, the instance when the target has a high-maneuvering movement often occurs. If we use the traditional methods to get the ISAR images, there will be many false scatters, and the quality of the images will be influenced. A signal decomposition method-adaptive chirplet decomposition is proposed in [5], this algorithm decomposes a multicomponent signals into the combination of a series of chirplet atoms. The parameters of the chirplet atoms are estimated adaptively by the principle of matching pursuit according to the characteristics of signals. This method improves the accuracy of the parameters estimation greatly, but the chirplet atoms still have the form of linear frequency-modulated signals and cannot characterize the complicated polynomial phase signals accurately.

In this paper, a new algorithm of signal decompositionmodified adaptive chirplet decomposition (MACD) is proposed. The form of the chirplet atoms is extended to the quadratic frequency-modulated signals in order to characterize the original signals more accurately. First, the definition of MACD is given, and the parameters estimation algorithm for monocomponent signal is studied, Then, the CLEAN technique is used to estimate the parameters of multicomponent signals. Finally, this method is used in the ISAR imaging of maneuvering targets, and the quality of ISAR images is improved greatly.

\section{THE BRIEF INTRODUCTION OF ADAPTIVE CHIRPLET DECOMPOSITION}

The adaptive chirplet decomposition is an approximation technique, providing representation of the signals, for a given signal $s(t)$, it can be represented by a sum of rectangle chirplet atoms $g_{n}(t)$ as follows:

$$
s(t)=\sum_{n=0}^{\infty} C_{n} g_{n}(t)
$$




$$
\begin{aligned}
g_{n}(t)=\left(\pi \sigma_{n}^{2}\right)^{-0.25} \exp \{ & -\frac{\left(t-t_{n}\right)^{2}}{2 \sigma_{n}^{2}}+j \omega_{n}\left(t-t_{n}\right) \\
& \left.+j \beta_{n}\left(t-t_{n}\right)^{2}\right\},
\end{aligned}
$$

where $\sigma_{n}, t_{n}, \omega_{n}$, and $\beta_{n}$ indicate time extent, time center, initial frequency, and chirp rate, respectively.

The chirplet atoms can be estimated based on the following principle:

$$
\left|C_{n}\right|^{2}=\max _{g_{n}}\left|\left\langle s_{n}(t), g_{n}(t)\right\rangle\right|^{2},
$$

where

$$
s_{n}(t)=s_{n-1}(t)-C_{n-1} g_{n-1}(t), \quad s_{0}(t)=s(t),
$$

where $s_{n}(t)$ is the residual of orthogonal projection of $s_{n-1}(t)$ on $g_{n-1}(t)$, which is

$$
s_{n}(t)=s(t)-\sum_{i=1, i \neq n}^{n-1} C_{i} g_{i}(t) .
$$

The decomposition of signals can be realized by computing (3)-(5).

Taking the Wigner-Ville distribution of the chirplet atom, (2) yields

$$
\begin{aligned}
\mathrm{WVD}_{g_{n}}(t, \omega)=2 \exp \{ & -\frac{\left(t-t_{n}\right)^{2}}{\sigma_{n}^{2}} \\
& \left.-4 \sigma_{n}^{2}\left[\frac{\left(\omega-\omega_{n}\right)}{2}-\beta_{n}\left(t-t_{n}\right)\right]^{2}\right\} .
\end{aligned}
$$

By calculating the partial derivative with respect to $\omega$ and by equaling it to zero, we can see that the Wigner-Ville distribution of the chirplet atom $g_{n}(t)$ is concentrated on the curve

$$
\omega=2 \beta_{n} t+\left(\omega_{n}-2 \beta_{n} t_{n}\right)=2 \beta_{n} t+\omega_{n},
$$

where $\omega_{n}=\omega_{n}-2 \beta_{n} t_{n}$.

\section{MODIFIED ADAPTIVE CHIRPLET DECOMPOSITION (MACD)}

The modified adaptive chirplet decomposition (MACD) is proposed based on the adaptive chirplet decomposition, and the atoms has the form of

$$
\begin{aligned}
h_{n}(t)=\left(\pi \sigma_{n}^{2}\right)^{-0.25} \exp \{ & -\frac{\left(t-t_{n}\right)^{2}}{2 \sigma_{n}^{2}}+j \omega_{n}\left(t-t_{n}\right) \\
& \left.+j \beta_{n}\left(t-t_{n}\right)^{2}+j \gamma_{n}\left(t-t_{n}\right)^{3}\right\},
\end{aligned}
$$

where $\sigma_{n}, t_{n}, \omega_{n}$, and $\beta_{n}$ indicate time extent, time center, initial frequency, and chirp rate, respectively. $\gamma_{n}$ is defined as the curvature of the atom, which makes the time frequency

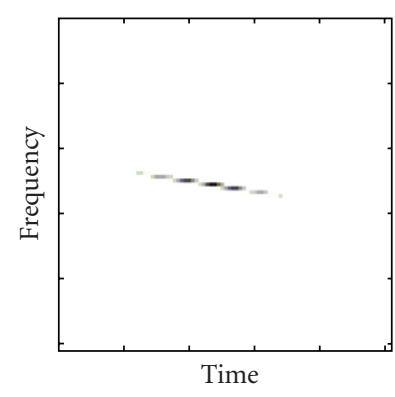

(a) Traditional chirplet atom

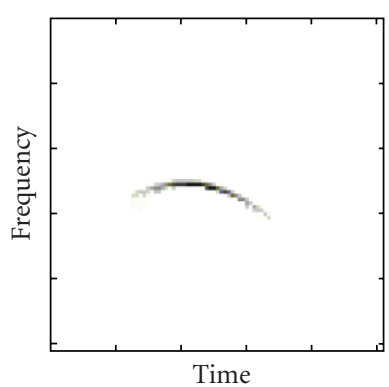

(b) MACD atom
Figure 1: Time frequency distributions of the atoms.

distribution of the atom become a curve, as shown in Figure 1.

The MACD is an approximation technique providing representation of the signals. For a given signal $s(t)$, it can be represented by a sum of new atom $h_{n}(t)$, that is,

$$
s(t)=\sum_{n=0}^{\infty} D_{n} h_{n}(t)
$$

The MACD algorithm finds the atom $h_{n}(t)$ most similar with $s_{n}(t):$

$$
\left|D_{n}\right|^{2}=\max _{h_{n}}\left|\left\langle s_{n}(t), h_{n}(t)\right\rangle\right|^{2}
$$

where

$$
s_{n}(t)=s_{n-1}(t)-D_{n-1} h_{n-1}(t),
$$

where $s_{n}(t)$ is the residual of orthogonal projection of $s_{n-1}(t)$ on $h_{n-1}(t), s_{0}(t)=s(t)$.

As we can see from (10) that the computation of the coefficient $D_{n}$ is a multidimensional nonlinear optimization problem, and we cannot get the analytic results. If we estimate the parameters by searching throughout the parameters space, the computation burden will be very heavy. Some algorithms for the parameters estimation are proposed for the linear frequency-modulated chirplet atoms $[6,7]$, but still need pursuit in a certain range or adaptive recursive computation. In this paper, a new practical parameters estimation algorithm of MACD is proposed.

\subsection{The parameters estimation of monocomponent signal}

For a monocomponent signal, we can use the parameters information and the characteristics of the modified chirplet atoms to get the estimation of the parameters by some mature methods in the signal processing. The steps are given as follows.

Step 1. Suppose the original signal $s(t)=D_{0} h_{0}(t)$, construct the reference signal $s_{\text {ref }}(t)=\exp \left(-j \gamma t^{3}-j \beta t^{2}\right)$, where $\gamma$ and 
$\beta$ are the variables to be determined. Multiply the reference signal with $s(t)$, we have

$$
\begin{aligned}
s(t) \times s_{\mathrm{ref}}(t)= & D_{0}\left(\pi \sigma_{0}^{2}\right)^{-0.25} \times \exp \left\{-\frac{\left(t-t_{0}\right)^{2}}{2 \sigma_{0}^{2}}\right\} \\
& \times \exp \left\{j\left(-\omega_{0} t_{0}+\beta_{0} t_{0}^{2}-\gamma_{0} t_{0}^{3}\right)\right\} \\
& \times \exp \left\{j\left(\omega_{0}-2 \beta_{0} t_{0}+3 \gamma_{0} t_{0}^{2}\right) t\right\} \\
& \times \exp \left\{j\left(\beta_{0}-\beta-3 \gamma_{0} t_{0}\right) t^{2}\right\} \\
& \times \exp \left\{j\left(\gamma_{0}-\gamma\right) t^{3}\right\} .
\end{aligned}
$$

We can see from (12) that when $\gamma=\gamma_{0}$ and $\beta=\beta_{0}-3 \gamma_{0} t_{0}$, the $s(t) \times s_{\text {ref }}(t)$ will be a sinusoidal signal of variable $t$. If we compute the Fourier transform of it, there will be a peak in the $\gamma-\beta$ plane, and the other undetermined parameters (such as $\omega_{0}, t_{0}$, and $\sigma_{0}$ ) will not influence it. Hence, we can get the estimated values $\hat{\gamma}_{0}$ and $\hat{\beta}$ by searching the peak of (12).

Step 2. Construct a new reference signal based on $\hat{\gamma}_{0}$ and $\hat{\beta}$ :

$$
\hat{s}_{\text {ref }}(t)=\exp \left\{-j \hat{\gamma}_{0} t^{3}-j \hat{\beta} t^{2}\right\} .
$$

Step 3. Compute the following equation:

$$
\widehat{\omega}=\max _{\widehat{\omega}}\left\{\operatorname{FFT}\left[s(t) \times \widehat{s}_{\text {ref }}(t)\right]\right\},
$$

where FFT $[\cdot]$ denotes the Fourier transform.

By designing a filter with narrow bandwidth and filtering out the narrow spectrum nearby $₫$, we can get an approximated sinusoid $u(t)$ by computing the inverse Fourier transform of the spectrum, that is,

$$
u(t)=\operatorname{IFFT}\left\{\operatorname{window}(\varpi) \times \operatorname{FFT}\left[s(t) \times \hat{s}_{\text {ref }}(t)\right]\right\},
$$

where

$$
\operatorname{window}(\omega)= \begin{cases}1, & \omega_{L}<\omega<\varpi_{R}, \\ 0, & \text { else }\end{cases}
$$

is the filter, $\omega_{L}$ and $\varpi_{R}$ are determined by the width of the spectrum. Hence, IFFT[ $\cdot]$ denotes the inverse Fourier transform. The width of the window will affect the result of signal decomposition, if the width is large, other component will be filtered out combined with the narrow spectrum and we cannot get an approximated sinusoid $u(t)$ by computing the inverse Fourier transform of it. Hence, the width of the window must be small. The precise implementation of the window width may be computationally expensive, but in the condition of ISAR imaging, the accuracy of the window width is not the main factor and it can be easily realized by the observation of the width of the narrow spectrum nearby $\varpi$. Compared with the Gaussian filter presented in [6], this method is more suitable to the ISAR imaging.

Step 4. Compute the Wigner-Ville distribution (WVD) of $u(t)$, we can get $\hat{t}_{0}$ of $s(t)$ as

$$
\widehat{t}_{0}=\arg \max _{t}\left[\mathrm{WVD}_{u}(t, \omega)\right] .
$$

We have to illustrate that the estimation of $\hat{t}_{0}$ based on (17) will produce error with low signal to-noise-ratio (SNR), and the derivation of (17) is based on the high SNR for the signals, which is appropriate for the ISAR imaging of maneuvering targets in most cases. Furthermore, the value of $\hat{t}_{0}$ will not influence the quality of the ISAR image greatly.

Then, we can get the estimated value of $\beta_{0}$ as

$$
\hat{\beta}_{0}=\hat{\beta}+3 \hat{\gamma}_{0} \hat{t}_{0} .
$$

Step 5. Construct the reference signal as

$$
s_{\text {ref }}^{\prime}(t)=\exp \left\{-j \hat{\beta}_{0}\left(t-\hat{t}_{0}\right)^{2}-j \hat{\gamma}_{0}\left(t-\hat{t}_{0}\right)^{3}\right\},
$$

and we can get the value $\hat{\omega}_{0}$ by

$$
\widehat{\omega}_{0}=\max _{\omega}\left\{\operatorname{FFT}\left[s(t) \times s_{\mathrm{ref}}^{\prime}(t)\right]\right\} .
$$

Step 6. Compute the value $\hat{\sigma}_{0}$ according to

$$
\widehat{\sigma}_{0}=\arg \max _{\sigma}|\langle s(t), h(t)\rangle|^{2},
$$

where

$$
\begin{aligned}
h(t)=\left(\pi \sigma^{2}\right)^{-0.25} \exp \{ & -\frac{\left(t-\hat{t}_{0}\right)^{2}}{2 \sigma^{2}}+j \widehat{\omega}_{0}\left(t-\hat{t}_{0}\right) \\
& \left.+j \hat{\beta}_{0}\left(t-\hat{t}_{0}\right)^{2}+j \hat{\gamma}_{0}\left(t-\hat{t}_{0}\right)^{3}\right\} .
\end{aligned}
$$

Step 7. Get the value $\hat{D}_{0}$ by (10).

It is obvious that the estimation of $\hat{D}_{0}$ will suffer from the error propagation problem. But, in this paper, the parameters estimation of MACD atoms can be treated as a choice of the initial values, which is accurate enough in the ISAR imaging. The method to improve the accuracy of the parameters estimation can be found in [8] as an example.

We estimate the parameters of monocomponent signal by the steps described above, since the process of computation requires only simple pursuit, Fourier transform, and multiplication, the computation efficiency is high, and the algorithm can be realized easily. Next, we will study the parameters estimation algorithm for the multicomponent signal.

\subsection{Parameters estimation of the multicomponent signal}

We use the CLEAN technique [9-11] to estimate the parameters of the multicomponent signal. The steps are given as follows.

Step 1. Estimate the curve gene $\hat{\gamma}_{1}$, chirp rate $\hat{\beta}_{1}$, time center $\hat{t}_{1}$, initial frequency $\hat{\omega}_{1}$, chirp width $\hat{\sigma}_{1}$, and the coefficient $\widehat{D}_{1}$ of the first signal $s_{1}(t)$ by (12), (17), (18), (20), (21), and (10).

Step 2. Subtract the estimated signal from the original signal according to

$$
s_{2}(t)=\operatorname{IFFT}\left\{[1-\operatorname{window}(\omega)] \times \operatorname{FFT}\left[s(t) \times e^{-j \hat{\phi}_{1}}\right]\right\} e^{j \hat{\phi}_{1}},
$$




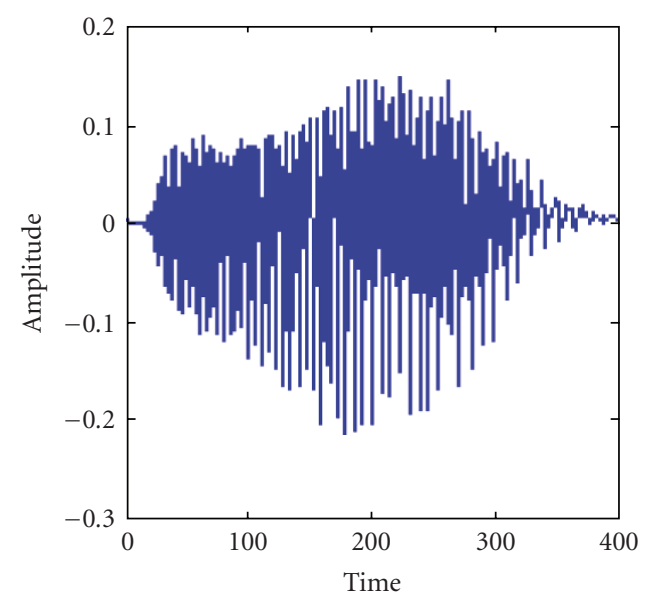

(a)

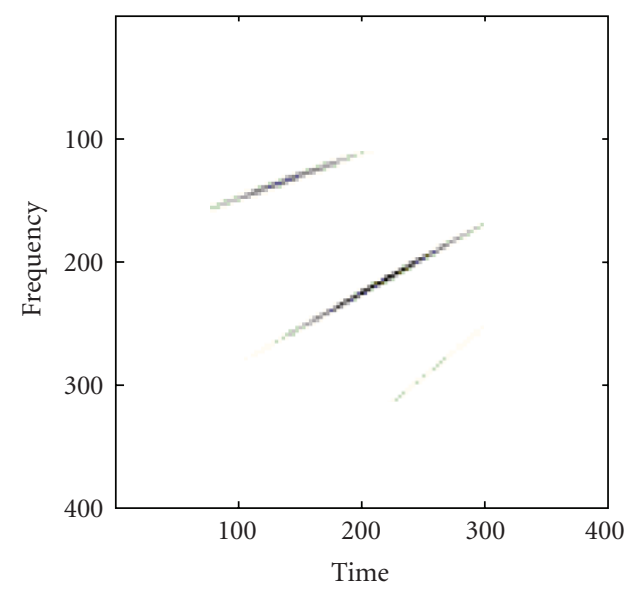

(c)

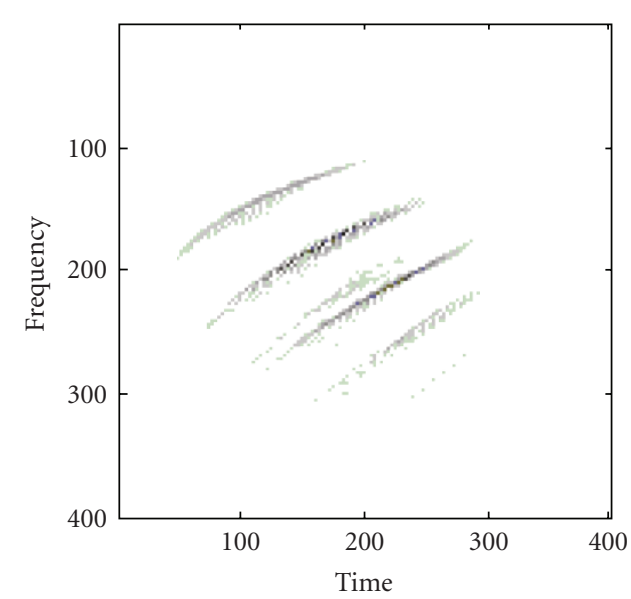

(b)

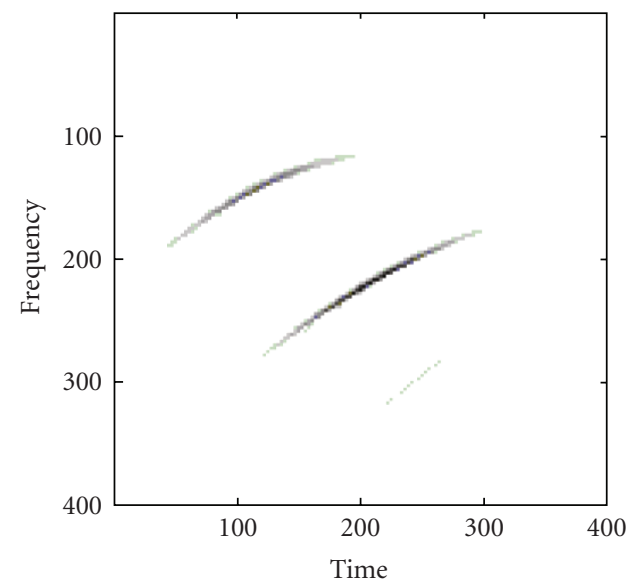

(d)

FIGURE 2: The bat signal and its time frequency distributions: (a) the bat signal, (b) Wigner-Ville distribution, (c) traditional chirplet decomposition, and (d) MACD algorithm.

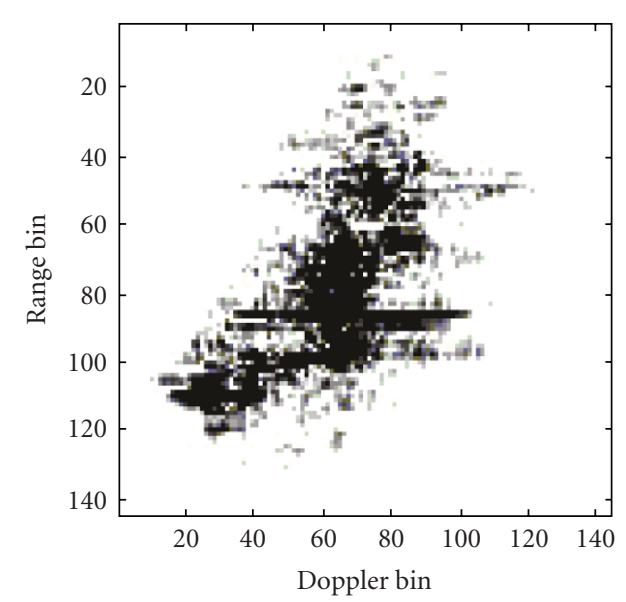

FIGURE 3: ISAR images of real data based on the range-Doppler algorithm.

where window $(\omega)$ is as (15), s(t) is the original signal, and

$$
\widehat{\phi}_{1}=\widehat{\omega}_{1}\left(t-\hat{t}_{1}\right)+\hat{\beta}_{1}\left(t-\hat{t}_{1}\right)^{2}+\hat{\gamma}_{1}\left(t-\hat{t}_{1}\right)^{3} \text {. }
$$

Step 3. Estimate the curve gene $\hat{\gamma}_{2}$, chirp rate $\hat{\beta}_{2}$, time center $\hat{t}_{2}$, initial frequency $\hat{\omega}_{2}$, chirp width $\hat{\sigma}_{2}$, and the coefficient $\hat{D}_{2}$ of the second signal $s_{2}(t)$ by (12), (17), (18), (20), (21), and (10).

Step 4. Repeat the steps above until the energy of the residual signal is less than a threshold $\varepsilon$ :

$$
\int_{-\infty}^{+\infty}\left|s_{\mathrm{res}}(t)\right|^{2} d t \leq \varepsilon .
$$

In practice, such as the ISAR imaging of maneuvering targets, the threshold $\varepsilon$ can be selected as about $10 \%$ of the energy for the original signal, which will be suitable to the ISAR imaging, and the selection of $\varepsilon$ is not the same in different circumstances.

We use the echolocation signals from brown bat to illustrate the effectiveness of the MACD algorithm, and the results are shown in Figure 2. Figure 2(a) is the bat signal in time domain, Figure $2(b)$ is the Wigner-Ville distribution of the signal, and the influence of the crossterms is obvious. Figure 2(c) is the result of the traditional 


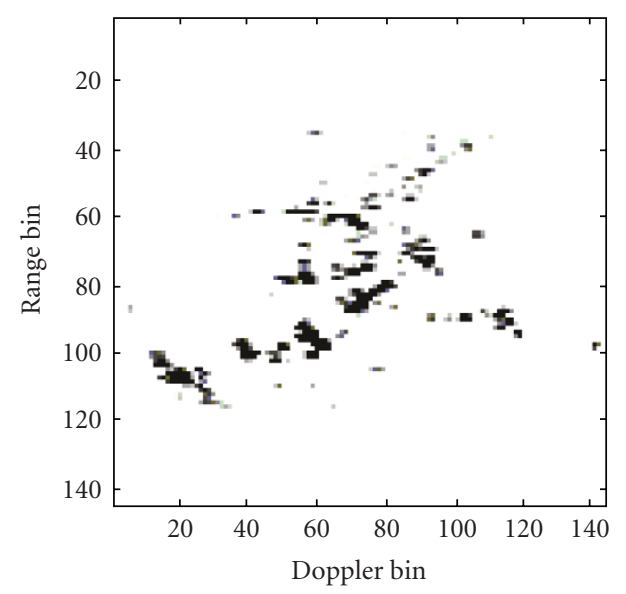

(a)

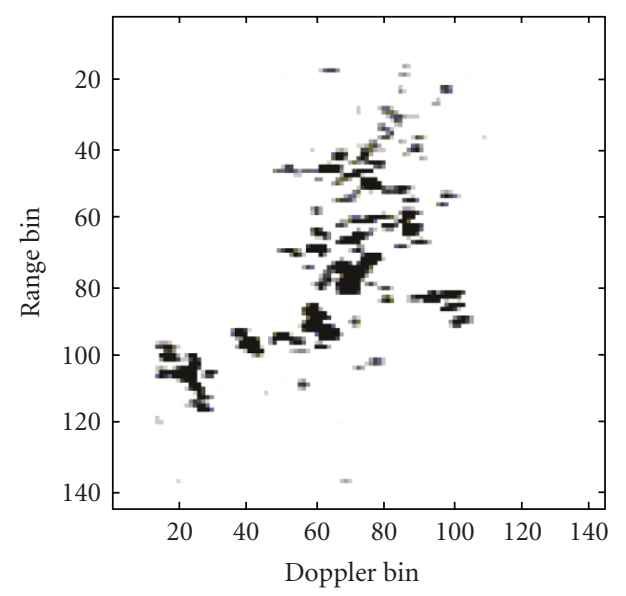

(c)

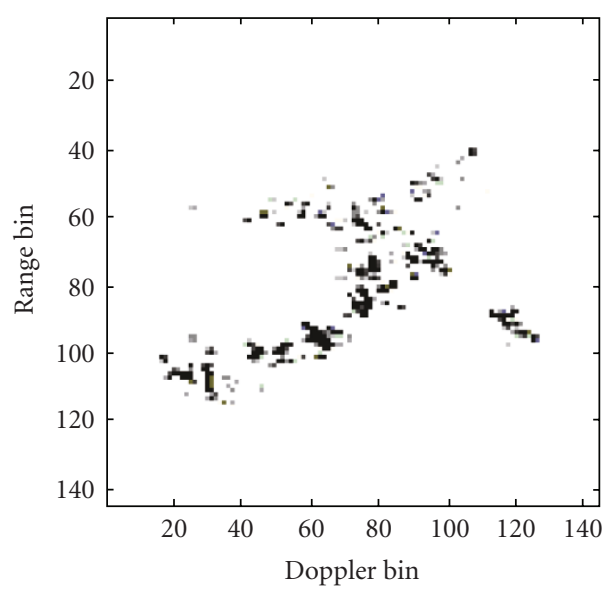

(b)

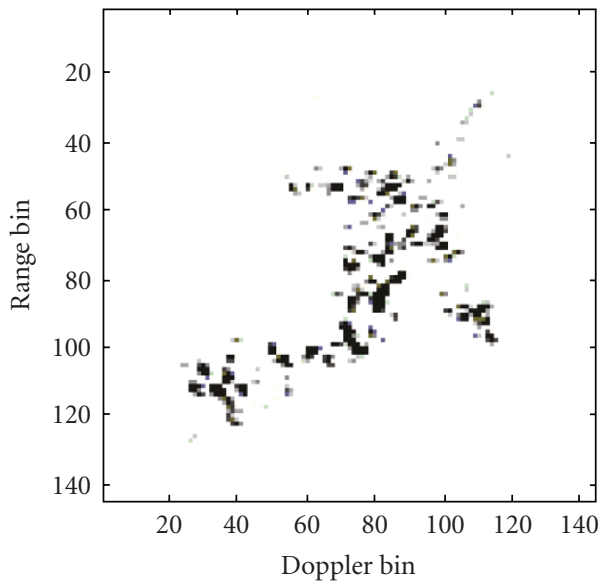

(d)

FIgURE 4: ISAR images of Yak-42: (a) chirplet decomposition, (b) MACD, (c) chirplet decomposition, and (d) MACD.

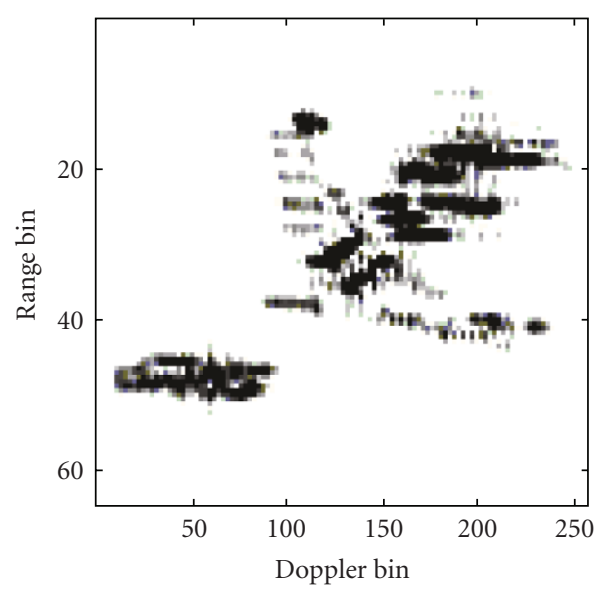

FIGURE 5: ISAR images of simulated data based on the rangeDoppler algorithm.

chirplet decomposition. The cross-terms have been reduced completely, but the nonlinear time varying property of the signal has not been characterized. Figure 2(d) is obtained by the MACD algorithm proposed in this paper, it is obvious that the MACD depicts the nonlinear and linear components of the bat signal and can demonstrate the time varying character of the signal more accurately.

\section{ISAR IMAGING OF MANEUVERING TARGETS BASED ON MACD}

When the target has a maneuvering movement, the rotation axis and velocity both vary with time. Hence, the received signals are time-varying and the traditional rangeDoppler algorithm will not be appropriate. Since the concept of range-instantaneous-Doppler algorithm was proposed recently, this algorithm is based on the assumption that the received signals of the scatters are multicomponent linear frequency-modulated signals. But, in some circumstances, the maneuverability of the target is high, and the received signals are high-order polynomial phase signals. In order to get the clear ISAR image in this case, a method based on the product high-order ambiguity function for multicomponent polynomial phase signals is proposed in [12], but this algorithm suffers from the computational burden. Another 


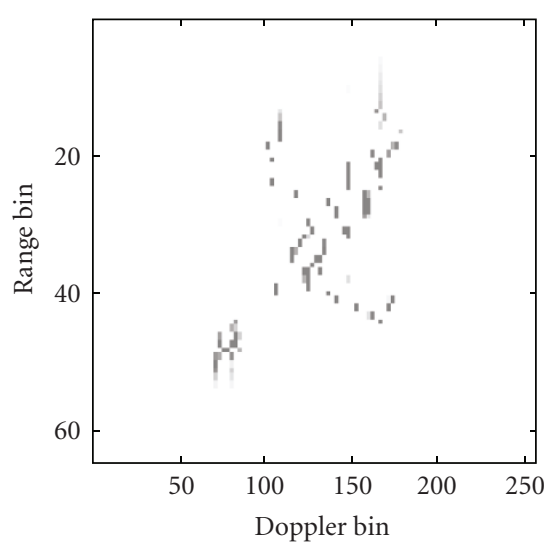

(a)

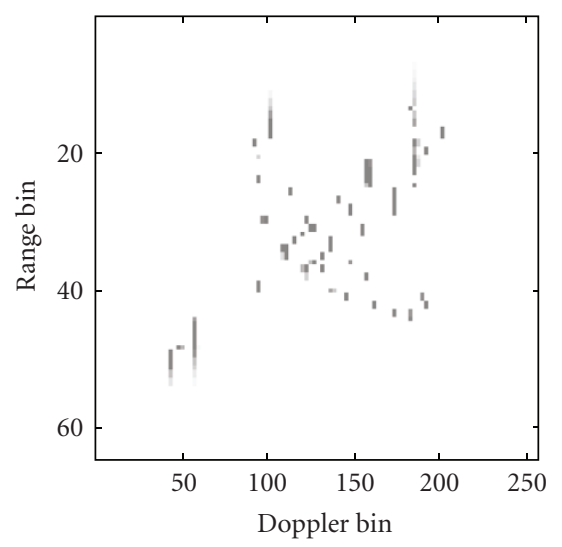

(d)

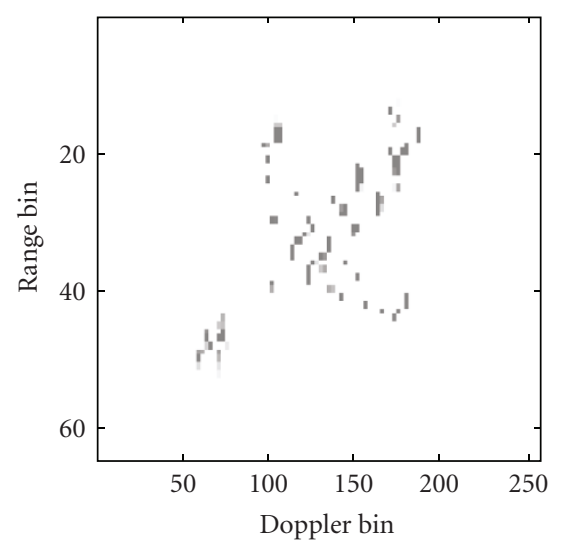

(b)

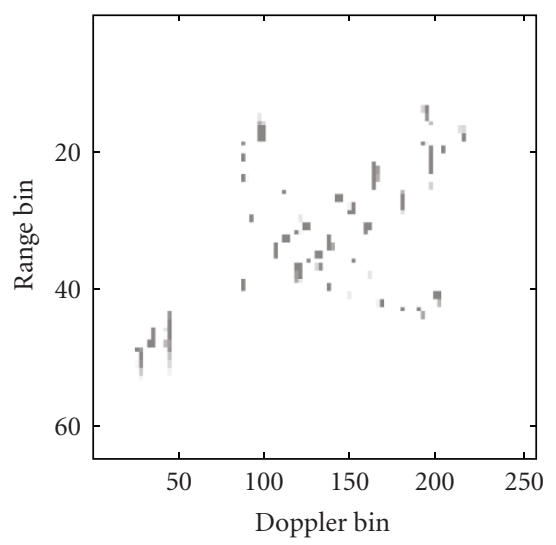

(e)

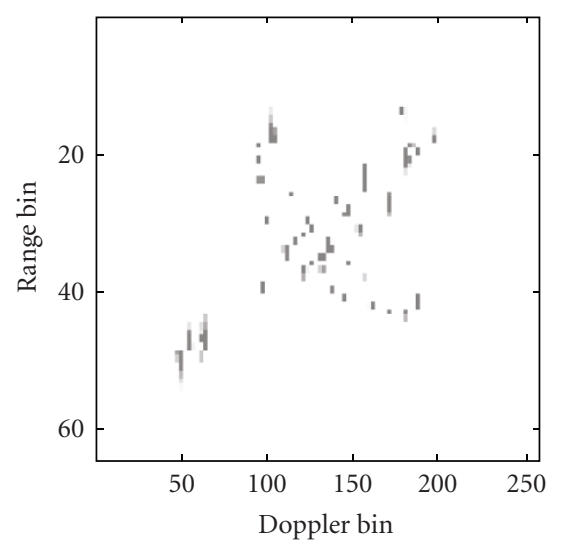

(c)

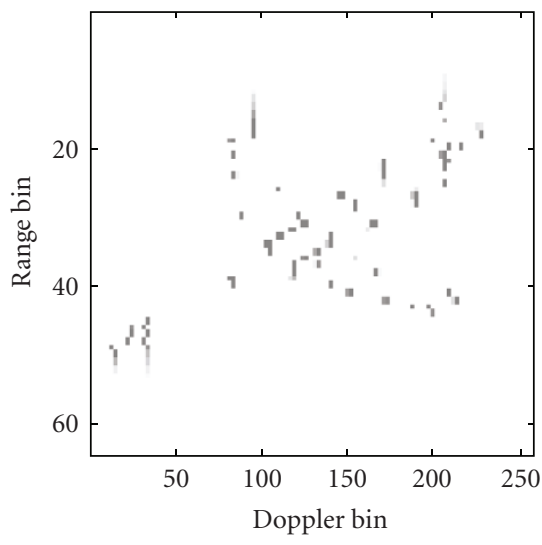

(f)

FIGURE 6: ISAR images of simulated data based on the chirplet decomposition.

algorithm based on the traditional chirplet decomposition is proposed in [13], this method approximates the received signals as a series of linear frequency modulated signals. But, the accuracy is not high, and there are many false scatters in the image. So, a new ISAR imaging algorithm based on MACD is proposed in this paper, and the quality of ISAR images is improved greatly.

After the motion compensation of the received signals, the received signals in a range bin can be approximated as multicomponent polynomial phase signals with changing amplitude, the model is as follows:

$$
\begin{aligned}
s(t) & =\sum_{k=1}^{K} a_{k}(t) \exp \left(j \phi_{k}(t)\right), \\
\phi_{k}(t) & =\phi_{0, k}+2 \pi \sum_{m=1}^{M} c_{k, m} t^{m},
\end{aligned}
$$

where $a_{k}(t)$ is the amplitude of the $k$ th signal, and $\varphi_{k}(t)$ is the phase.

As mentioned previously, the MACD algorithm can characterize the multicomponent polynomial phase signals more accurately than the traditional chirplet decomposition. Now, we compare the ISAR imaging results of real data and simulated data based on the two algorithms.

\section{(1) Real data}

We choose a segment of real data when the Yak-42 plane has a high-maneuvering flight. The radar works in the $\mathrm{C}$ band, with a bandwidth of $400 \mathrm{MHz}$. Figure 3 is the ISAR image based on the range-Doppler algorithm; the image is blurred and cannot be recognized. Figures $4(\mathrm{a})$ and $4(\mathrm{c})$ are the ISAR images based on the traditional chirplet decomposition at different time, and the quality is improved, the change of the target's motion is also reflected. Figures $4(\mathrm{~b})$ and $4(\mathrm{~d})$ are the ISAR images based on the MACD algorithm at the same time with Figures 4(a) and 4(c), we can see that the quality is improved furthermore, and the false scatters are decreased. In both cases, we decompose the received signals of each range bin into three chirplet atoms or MACD atoms, and the energy of the residual signals is less than $10 \%$ of the original signals, that is, the energy threshold can be chosen as $10 \%$.

\section{(2) Simulated data}

We choose the simulated data of B727 plane, which has a slight maneuvering flight. The radar transmits stepped frequency signals, the carrier frequency $f_{0}=9 \mathrm{GHz}$, bandwidth $B_{d}=150 \mathrm{MHz}$, the number of pulses is 256 . 


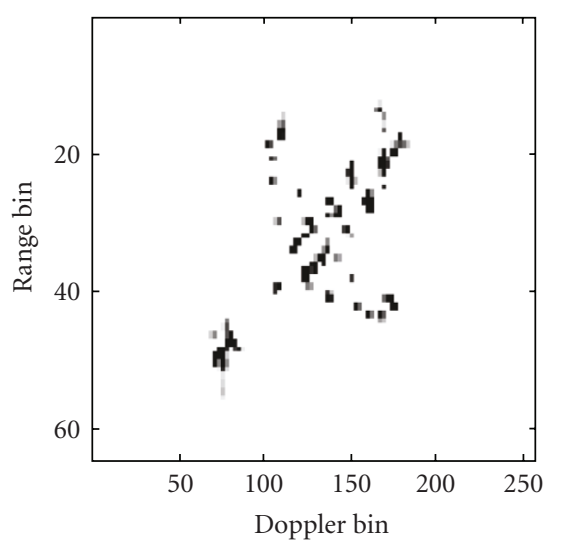

(a)

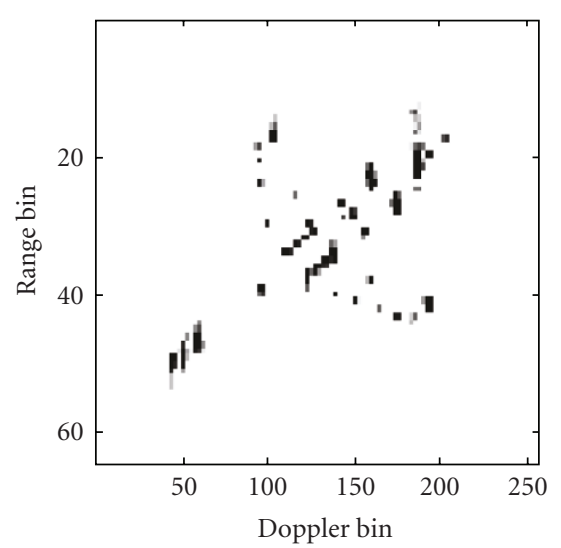

(d)

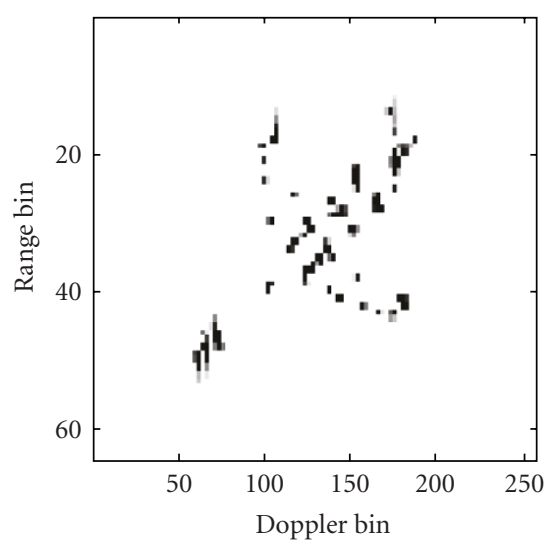

(b)

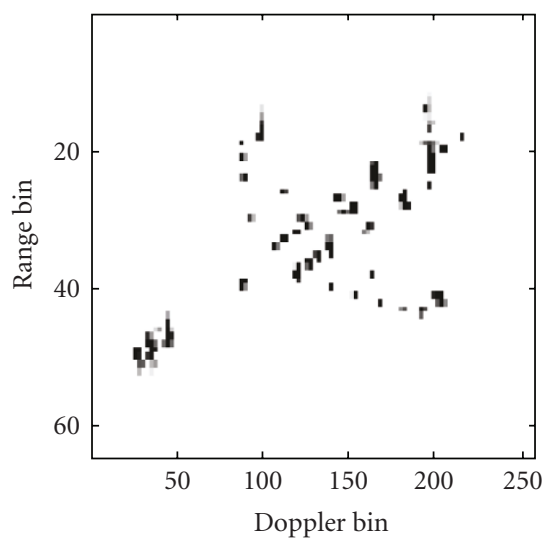

(e)

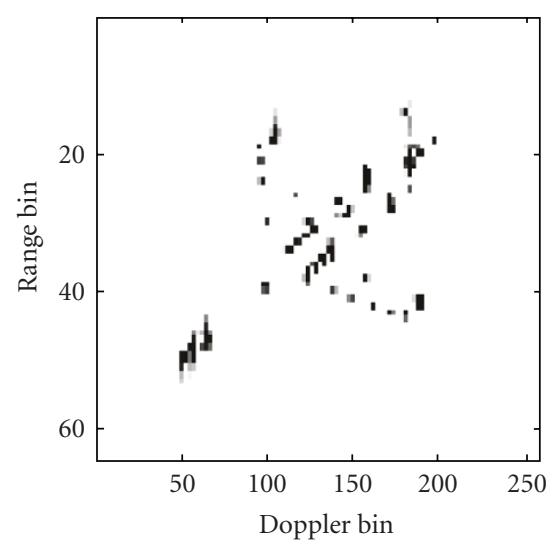

(c)

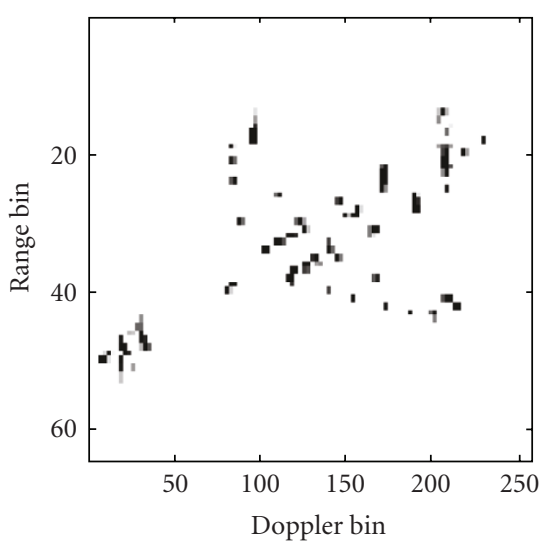

(f)

FIGURE 7: ISAR images of simulated data based on the MACD algorithm.

Figure 5 is the ISAR image based on the range-Doppler algorithm, the image is blurred but the shape of the plane can be recognized for the slight maneuverability. Figures 6(a)6(f) are the ISAR images based on the traditional chirplet decomposition at different time, and the quality is improved greatly. Figures $7(a)-7(f)$ are the ISAR images based on the MACD algorithm at the same time with Figures 6(a)-6(f). In both cases, we decompose the received signals of each range bin into two chirplet atoms or MACD atoms. For the slight maneuverability of the targets, the quality of the images based on the MACD algorithm is analogous with or a bit better than the images based on the traditional chirplet decomposition, which indicates the robustness of the proposed algorithm.

From the two examples above, we can see that the MACD algorithm can generate clear instantaneous ISAR images for common maneuvering targets. In the condition of fast maneuvering movements, the long coherent processing intervals (CPIs) will be used to obtain finer resolutions, and the image plane is nonstationary. This causes severe defocusing which cannot be corrected by using higher-order polynomial phase models, and this is the main limitation for the technique in this paper. An efficient way to solve this problem is the subaperture and super-resolution techniques.

\section{CONCLUSION}

A new algorithm of signal decomposition-modified adaptive chirplet decomposition (MACD) is proposed in this paper. The traditional chirplet decomposition cannot characterize the original signal accurately, and this disadvantage is overcome by extending the chirplet atom to the form of quadratic frequency-modulated signal. Then, the parameters estimation algorithm of monocomponent atom is given, and the CLEAN technique is used in order to estimate the parameters of multicomponent signals. Finally, we use this method in the ISAR imaging of maneuvering targets, and the quality of ISAR images improves greatly.

\section{REFERENCES}

[1] Z. Bao, G. Wang, and L. Luo, "Inverse synthetic aperture radar imaging of maneuvering targets," Optical Engineering, vol. 37, no. 5, pp. 1582-1588, 1998.

[2] V. C. Chen and S. Qian, "Joint time-frequency transform for radar range-Doppler imaging," IEEE Transactions on Aerospace and Electronic Systems, vol. 34, no. 2, pp. 486-499, 1998.

[3] P. T. Gough, "A fast spectral estimation algorithm based on the FFT," IEEE Transactions on Signal Processing, vol. 42, no. 6, pp. 1317-1322, 1994. 
[4] J. Li and P. Stoica, "Efficient mixed-spectrum estimation with applications to target feature extraction," IEEE Transactions on Signal Processing, vol. 44, no. 2, pp. 281-295, 1996.

[5] S. Mann and S. Haykin, "The chirplet transform: physical considerations," IEEE Transactions on Signal Processing, vol. 43, no. 11, pp. 2745-2761, 1995.

[6] H. Zou and Z. Bao, "An efficient algorithm for adaptive Chirplet-based signal decomposition," Acta Electronica Sinica, vol. 29, no. 4, pp. 515-517, 2001.

[7] Q. Y. Yin, Z. F. Ni, S. Qian, and D. Chen, "Adaptive oriented orthogonal projective decomposition," Acta Electronica Sinica, vol. 25, no. 4, pp. 52-58, 1997.

[8] Q. Yin, S. Qian, and A. Feng, "A fast refinement for adaptive Gaussian chirplet decomposition," IEEE Transactions on Signal Processing, vol. 50, no. 6, pp. 1298-1306, 2002.

[9] J. Tsao and B. D. Steinberg, "Reduction of sidelobe and speckle artifacts in microwave imaging: the CLEAN technique," IEEE Transactions on Antennas and Propagation, vol. 36, no. 4, pp. 543-556, 1988.

[10] M. Martorella, N. Acito, and F. Berizzi, "Statistical CLEAN technique for ISAR imaging," IEEE Transactions on Geoscience and Remote Sensing, vol. 45, no. 11, pp. 3552-3560, 2007.

[11] I.-S. Choi and H.-T. Kim, "Two-dimensional evolutionary programming-based CLEAN," IEEE Transactions on Aerospace and Electronic Systems, vol. 39, no. 1, pp. 373-382, 2003.

[12] Y. M. Zheng, M. D. Xing, and Z. Bao, "Imaging of maneuvering targets based on parameter estimation of multicomponent polynomial signals," Journal of Xidian University, vol. 27, no. 4, pp. 471-475, 2000.

[13] G. Y. Wang and Z. Bao, "ISAR imaging based on Chirplet decomposition to maneuvering targets," Acta Electronica Sinica, vol. 27, no. 3, pp. 29-31, 1999. 